\title{
Sociedad del conocimiento y la economía
}

\section{Knowledge society and the economy}

Fabián Terán-Cano

Universidad Iberoamericana del Ecuador-UNIB.E, Ecuador

Autor para correspondencia: fabianteran@ hotmail.com

Fecha de recepción: 16 de Noviembre de 2017 - Fecha de aceptación: 15 de Mayo de 2018

Resumen: La principal contribución de este artículo es la caracterización de la sociedad del conocimiento, sus orígenes e interrelaciones, y su tránsito hacia lo que se denomina como nueva economía, lo que quiere decir que no solamente se produce bienes que buscan una renta, sino que produce ideas que permiten a la luz de este nuevo conocimiento e innovación, transformar las actividades productivas para alcanzar el desarrollo económico superando los problemas permanentes de injusticia e inequidad. En el documento se realiza una contextualización delo que es la sociedad del conocimiento, destacando los aspectos conceptuales relacionados con la innovación tecnológica y la economía del conocimiento, ubicando al final un esbozo del caso ecuatoriano en el escenario de la globalización. Se realiza un análisis de la economía del conocimiento o nueva economía que está relacionada con los avances tecnológicos que proporciona la informática a través de las redes de comunicación que se desarrollan como parte del denominado proceso de globalización, y se relaciona con los avances alcanzados en el Ecuador como parte de los objetivos del Plan Nacional de Buen Vivir, y de la propia interacción de la economía con el mundo.

Palabras Claves: sociedad del conocimiento; economía; sociedad; innovación; globalización; bien público

\begin{abstract}
The main contribution of this article is the characterization of the knowledge society, its origins and interrelationships, and its transit towards what is called new economy, which means that not only goods that look for an income are produced; but it produces ideas that based on this new knowledge and innovation allow to transform the productive ideas to reach the economic development by overcoming the permanent problems of justice and inequality. Moreover, in this document it is carried out the contextualization of the knowledge society meaning through the highlighting of the conceptual aspects related to technology innovation and the economy knowledge, by means of placing an outline of the Ecuadorian case in the globalization stage at the end. An analysis is made of the knowledge economy or new economy that is related to the technological advances that information technology provides through the communication networks that are developed as part of the so-called globalization process, and is related to the progress made in the Ecuador as part of the objectives of the National Plan for Good Living, and the very interaction of the economy with the world.
\end{abstract}

Key Words: knowledge society; economy; society; innovation; globalization; public good 


\section{Introducción}

\section{La Sociedad del conocimiento}

Hoy en día hablar de la sociedad del conocimiento significa estar al día de una de las discusiones estelares que se realizan en las ciencias sociales, que de alguna manera resume las transformaciones sociales que se producen en la sociedad. Se trata de un concepto que resume los cambios sociales que se están produciendo en la sociedad moderna y sirve para el análisis de estas transformaciones. (Krüger, K. 2006).

La noción sociedad de conocimiento tiene sus orígenes en los años 1960 cuando se estudió el comportamiento de las sociedades industriales y partir de ello se empieza a considerar la noción de la sociedad post-industrial con la presencia activa de una nueva capa social de trabajadores que se encaminaban hacia una sociedad de conocimiento. Este tipo de sociedad está caracterizada por una estructura económica y social, en la que el conocimiento ha substituido al trabajo, a las materias primas y al capital como fuente más importante de la productividad, crecimiento y desigualdades sociales. (Drucker, P. 1994).

La denominación de sociedad del conocimiento expresa la transición de una economía que produce productos a una economía basada en servicios, que para el efecto demanda de profesionales altamente calificados, donde el conocimiento, la generación de ideas se convierten en la principal fuente de innovación y desarrollo de nueva tecnología que demandan las economías emergentes como la principal fuente de productividad, lo que necesariamente demanda contar con un sistema educativo de calidad, evaluado y acreditado para potenciar la transición hacia la sociedad del conocimiento.

La globalización de la economía, a partir del tránsito de una economía nacional a regional y mundial, generó grandes cambios en los procesos económicos que han creado procesos multidimensionales con amplias repercusiones en las políticas comerciales y económicas y sus consecuentes implicaciones en los mercados de productos y de servicios que se traducen en la necesidad de expansión de las empresas multinacionales para aprovechar las nuevas oportunidades de integración de mercados; la producción e intercambio multinacional; competencia de las multinacionales por los mercados; desarrollo de procesos de innovación y científica tecnológica que han significado el surgimiento de nuevas demandas para las organizaciones y su necesaria adaptación a los cambios del entorno. En ese contexto, se ha reconocido al conocimiento como parte del valor del producto, como un relevante factor de producción, inclusive por sobre el trabajo industrial o manual. (UNESCO, 2005).

En los actuales momentos, los cambios y transformaciones son incesantes, motivados por una creciente globalización, donde la universidad no puede estar inmutable a esta realidad y las demandas sociales, de allí que es menester contar con un sistema universitario de calidad, que potencie investigaciones pertinentes y alineadas a la planificación central con la finalidad de responder funcional y oportunamente a las nuevas y exigentes demandas que se presentan.

La economía entonces tiene una estrecha relación con la sociedad del conocimiento, y el nuevo paradigma tecnológico al que asistimos está basado en la economía, y ésta a su vez con la educación, que permite mediante la participación de profesionales calificados transformar los procesos productivos para hacerlos más eficientes, donde el vínculo entre la entre universidad, 
estado y el sector productivo debe potenciarse, para poder participar en la creciente competencia internacional, la demanda de creatividad y de progreso técnico que será cada vez mayor; lo que posibilitará mejorar las condiciones de vida de la población, eliminar la pobreza extrema, el analfabetismo, la falta de salud, la inseguridad, como medio para alcanzar el bienestar de los ciudadanos, y con ello catapultar una nueva economía que promueva relaciones de intercambio de mercancías más allá de los aranceles y productos, lo que implica adaptarse a nuevos contextos, culturas y a la velocidad con la que se transmiten los recursos e intercambios que hacen del mundo cada vez más cercano.

\section{Método}

La presente investigación se realiza en base a un amplio análisis bibliográfico sobre la temática que ocupa el artículo, abordando las relaciones existentes entre la denominada sociedad del conocimiento y su repercusión en la economía, aspectos que coadyuvan al debate en la búsqueda de una sociedad más equitativa y de un desarrollo económico sostenible en beneficio de la población.

\section{Resultados y discusión}

A partir de varias investigaciones, se planteó que los sectores relacionados con los productos intangibles como los financieros e informáticos, representaban ingresos superiores, en relación con los procesos inherentes a la industria basados en la manufactura. Desde los años setenta y ochenta, se ha venido teorizando sobre el constructo sociedad del conocimiento. Peter Drucker (1993), citado por Darín, S.; González, Y. (2008), afirma lo siguiente sobre la sociedad del conocimiento:

"La productividad del saber va a ser cada vez más el factor determinante en la posición competitiva de un país, una industria una empresa. Respecto del saber, ningún país, ninguna industria, ninguna empresa, tiene ventajas o desventajas naturales. La única ventaja que puede tener es respecto de cuánto obtiene del saber disponible para todos. Lo único que importará cada vez más en la economía nacional e internacional serán los resultados que consiga la productividad del saber". (p. 55).

Se ha discutido y se sigue discutiendo ampliamente en el campo de las ciencias sociales este concepto y se han planteado múltiples visiones para definir la sociedad del conocimiento. No obstante, de manera general la llamada sociedad del conocimiento y la información ha sido utilizada para describir el tránsito de la era industrial a la era de la información, cuyos elementos limitantes lo han constituido el capital financiero o recurso tangible y los activos intangibles, respectivamente. (Pérez, Núñez, \& Font, 2016).

Peter Drucker expresó que la fuerza laboral enfrentaba un gran reto, dado que el mercado de trabajo demandaría de trabajadores con mayores niveles de educación que sean capaces de procesar grandes volúmenes de información, sistematizarla y a partir de aquello desarrollar conocimiento, siendo este factor un elemento relevante en la productividad y por tanto en el desarrollo de la economía. (Drucker, P. 1969)

Se puede señalar que los cambios económicos en los sectores de la producción de bienes disminuyen su prevalencia en relación al sector de servicios, dado que su expansión basada en el 
conocimiento demanda de una mayor cualificación académica y profesional. En la sociedad del conocimiento, la tendencia es valorar más el conocimiento en los diferentes ámbitos que las materias primas y el capital, como ocurría en la sociedad industrial (Drucker, 1994). En los años noventa se profundizó en el concepto de sociedad del conocimiento definiéndose como la capacidad de utilizar o generar el saber para innovar constantemente el conjunto de las actividades humanas. (Stehr, N. 1994).

La sociedad del conocimiento "hace especialmente hincapié en la capacidad para producir e integrar nuevos conocimientos y acceder a la información, el conocimiento, los datos y una vasta gama de conocimientos prácticos". (UNESCO, 2005, p.230). Los cambios en la estructura económica y por tanto en los mercados laborales, han sustituido el trabajo, materias primas y capital, por el conocimiento como una de las fuentes más importantes para elevar la productividad y crecimiento económico, pero también las desigualdades sociales. (Nino, E. 2011).

Se plantea entonces que el conocimiento es el nuevo y más relevante factor de la producción, inclusive por sobre el trabajo industrial o manufacturero. Ciertos enfoques sobre la sociedad del conocimiento han señalado que el concepto de sociedad del conocimiento se relaciona con ingenuidad social, mientras que el concepto economía del conocimiento se relaciona con valor de cambio, por lo que desde esa perspectiva la economía del conocimiento busca el bien privado, y la sociedad del conocimiento el bien público. (Hargreaves, A. 2003) .

Esta visión se asocia a visiones económicas capitalistas enmarcadas en términos de productividad y no se asocia en elementos de transformación social. Otros enfoques centran a la sociedad del conocimiento desde el punto de vista de la conformación de comunidades de ciudadanos que trabajen de manera colaborativa que gestionen, construyan y apliquen el conocimiento en la resolución de problemas locales con una visión global con sentido crítico y ético apoyado en las tecnologías de la información y comunicación. (Tobón, Guzmán, \& Cardona, 2015).

Según (Bizzozero, 2015) el crecimiento económico de los países emergentes, está generando algunas tendencias que configuran cambios en la educación, investigación e innovación. La sociedad del conocimiento en el mundo educativo implica un nuevo enfoque en la formación, de tal manera que mediante procesos colaborativos se innoven los procesos educativos y fortalecer la formación del pensamiento crítico. En el mundo de las organizaciones, la sociedad del conocimiento pretende el establecimiento del aprendizaje continuo, a partir del conocimiento generado en las organizaciones mediante el trabajo colaborativo que permitan alcanzar los objetivos organizacionales. (Tobón et al., 2015).

\section{La economía del conocimiento}

La Economía del Conocimiento se la concibe como el desplazamiento de la producción de bienes a la producción de ideas, lo que supone el tratamiento de información. Castells (2001), citado por Guevara (2013), señala que la Economía del Conocimiento no se basa sólo sobre el conocimiento. "De hecho, presenta tres características claves, las cuales se basan, en última instancia, en la micro-tecnología: 
1. Productividad en información, apoyada a su vez en las tecnologías de la información (TICs);

2. Tiempo real; conectividad global de flujos de capital, productividad, y gestión (no sólo referida al transporte internacional de mercancías), lo que sólo es factible gracias a la infraestructura tecnológica (Internet).

3. Trabajo en red, o Networking; Internet está en el corazón de estas redes, pero es mucho más que una tecnología. La Economía del Conocimiento no es la economía de los que proveen Internet, sino la de quienes la usan”. (Guevara, C. 2013, p. 13).

De acuerdo a Castells, la Economía del Conocimiento podría formular y establecer sus propias reglas sobre el capital, la fuerza de trabajo, y la gestión y está fundamentalmente basada en la capacidad de innovación, sea esta administrativa, tecnológica, social y política; que tiene como esencia a la información, al conocimiento, y donde las TICs son instrumentos necesarios y fundamentales para alcanzar una sociedad más equitativa, que promueva un desarrollo económico para alcanzar mejorar la calidad de vida de la población.

La nueva economía del conocimiento es el resultado de la incorporación y progreso de la ciencia y, como tal, se encuentra en plena fase de construcción. El conocimiento ha sido siempre un factor de producción asociado al capital humano; no obstante en la actualidad, la capacidad para administrar, almacenar y transmitir grandes cantidades de información a bajo precio es un elemento central de los procesos organizativos y de la sociedad. De acuerdo a Drucker (1994), en el futuro próximo, no existirán países pobres, sino sólo países incapaces de crear, adquirir y/o aplicar el conocimiento. (Lamo de Espinosa, 2004).

En la nueva economía la tradicional dicotomía capital y trabajo es superada y no constituye el problema central de la generación de la riqueza. Ciertamente, tanto capital como trabajo son requeridos para la producción de bienes, pero el conocimiento es la base o esencia de la economía. La innovación y la productividad son expresiones concretas del conocimiento individual y del conocimiento organizativo y, en tal perspectiva, la gestión del conocimiento, donde el rol de las tecnologías de la información y conocimiento es clave, constituye una tarea esencial para el éxito de las organizaciones en la nueva economía. La nueva economía no se sustenta en la producción de objetos o cosas, sino que esencialmente en la producción de ideas e intangibles, tales como: innovaciones, patentes, sistemas de organización, "know how", y en la capacidad de emplear el conocimiento para generar valor funcional, pero sobre todo para crear valor estratégico, para lo cual requiere recursos humanos capacitados, industrias de alta tecnología, equipos de comunicación que sean utilizados por los sectores productivos en su proceso de producción. En la nueva economía, si bien las industrias líderes son las de software, telecomunicaciones, es decir, las intensivas en conocimiento, todas las organizaciones tienen posibilidad de crear valor estratégico y ser exitosas en la medida que sean capaces de gestionar el conocimiento. (Rodríguez, E.; Palma, A. 2010).

\section{Conocimiento e innovación tecnológica}

El conocimiento y la innovación tecnológica tienen un papel fundamental en las actividades económicas que realizan los diferentes agentes económicos en la economía y en el desarrollo de las naciones. Si bien hoy se habla de globalización del conocimiento, esto no ocurre en la realidad a pesar de que la mayoría delos países tienen una economía de libre 
mercado abierta al mundo, dado que las tecnologías de la información y las comunicaciones no son bienes públicos, su acceso es limitado y no tienen la universalidad que se pregona, produciéndose altos niveles de dependencia que obligan a los países menos desarrollados a reorientar sus esfuerzos a la innovación

El conocimiento económico y la innovación tecnológica juegan un papel capital en la política económica que busca el desarrollo económico, y su globalización es considerada una de las manifestaciones de la nueva economía que está basada en el conocimiento, en la cual estaría inmersa la denominada sociedad del conocimiento y cuyo motor lo constituyen las Tecnologías de la Información y las Comunicaciones (TICs). (Rúa, N. 2006).

A pesar de un importante avance sobre todo en las TICs, y al acceso de un segmento importante de la población, en el uso de la telefonía móvil y el internet, la globalización, la interacción de los mercados, la disponibilidad de los recursos no es lo que en la realidad sucede, y es más evidente la brecha entre las sociedades que ha sido agudizada por la globalización, que pone de manifiesto la existencia personas, empresas, instituciones, regiones y sociedades que poseen las condiciones materiales y culturales para operar en el mundo digital y los que no pueden o no quieren adaptarse a la velocidad del cambio. (Castells, M.1999).

El desarrollo científico-tecnológico, en razón de que no es tratado como bien público, debe enfocarse en términos del desarrollo de los países, pero por la vía de la creación y apropiación de conocimiento, a la medida de sus necesidades, y nunca con la pretensión de cerrar la brecha en relación con los países desarrollados; sin embargo el conocimiento científicotecnológico, y en especial el tecnológico, si debe considerarse como un bien público cuando es susceptible de universalización, esto es, cuando cumple las condiciones básicas de libre acceso, circulación sin restricciones y difusión en todos los niveles en beneficio de la sociedad y como motor del crecimiento económico.

Los países menos desarrollados se ven abocados a actuar dentro de la realidad de la globalización y de la revolución tecnológica y a dotarse de capacidad de reflexión, crítica y transformación social y política, y de conocimiento e información con el fin de poder confrontar las poderosas fuerzas que tienden a excluirlos de sus posibilidades de desarrollo y a generar fuertes condiciones de dependencia, o interdependencia. Así, un país menos desarrollado tiene que decidir si hace I+D (Investigación y Desarrollo) en tecnologías de punta o emergentes o si opta por hacer apropiación, adaptación, reconversión, licenciamiento, etc. de tecnología, a la medida de sus necesidades. A nivel mundial, la producción científico-tecnológica se globaliza mediante los medios de comunicación, aprovechando las comunicaciones para difundir su producción científica.

En la nueva economía, la información y el conocimiento pasan a ser una nueva forma de riqueza y poder. Si se asume el conocimiento como un bien mercancía se somete a las reglas del juego de la economía de mercado, donde las leyes de oferta y demanda son las que interactúan, pero esto no posibilita que adquiera la categoría de público, en parte por razones de costos y, otra por razones de barreras asociadas a derechos. Si no es un bien público, no cumple con la condición central de la universalización del conocimiento. (Finquelievich, S. 2004). 


\section{Hacia una nueva economía en Ecuador}

En el Ecuador, el Plan Nacional de Desarrollo 2013-2017, propone un cambio paulatino hacia la sociedad del conocimiento, es decir pasar de una economía basada en la producción de bienes finitos a una economía de bienes infinitos, vinculándose a la universalidad, aprovechando las nuevas tecnologías de la información y la comunicación como factor para el desarrollo de las capacidades para actuar en el mundo globalizado.

La economía ecuatoriana se ha caracterizado por ser proveedora de materias primas en el mercado internacional y al mismo tiempo importadora de bienes y servicios elaborados. Los constantes e imprevistos cambios en los precios internacionales de las materias primas, así como su creciente diferencia frente a los precios de los productos de mayor valor agregado y alta tecnología, han colocado a la economía ecuatoriana en una situación de intercambio desigual sujeta a los vaivenes del mercado mundial, de allí que en los últimos diez años se ha impulsado un cambio del patrón de especialización productiva de la economía que le permita al Ecuador generar mayor valor agregado a su producción en el marco de la construcción de una sociedad del conocimiento. Transformar la matriz productiva constituye uno de los retos más ambiciosos del país, que de llegar a concretarse permitirá al Ecuador superar el actual modelo de generación de riquezas, por un modelo basado en el conocimiento y las capacidades los ecuatorianos.

En esta dirección el gobierno ecuatoriano en los últimos diez años, propició la creación de tres universidades emblemáticas, cuyo modelo de universidad se basa en la producción de conocimiento, con un énfasis en la investigación tecno-científica, alineada a los negocios y la economía del conocimiento, que debe ser productiva y eficiente, altamente competitiva, emprendedora, cuyo referente principal es la Universidad de Investigación de Tecnología Experimental Yachay, creada con el objetivo de llevar a cabo la investigación básica y de esta manera proveer el conocimiento; sin embargo, este loable propósito no se ha cumplido hasta el momento a pesar de grandes inversiones en la construcción de la denominada ciudad del conocimiento y dista mucho en su ejecución de ser una entidad generadora de conocimiento e innovación para ser protagonista de una nueva economía. (Villavicencio, A. 2013).

La nueva economía que permita un crecimiento económico como una vía para el progreso no será exclusivamente la gran empresas, sino un nuevo actor social que constituyen los emprendedores, que son los nuevos agentes económicos que conduzcan la actividad empresarial, más cuando la micro, pequeña y mediana empresa han tenido un impacto significativo en el desarrollo económico del Ecuador, es por ello que las micro finanzas conforman un sector reconocido por instituciones nacionales y organismos internacionales; lo cual será posible incorporando las TICs, que facilitan las comunicaciones, el control y la velocidad de los procesos.

Por otro lado, si bien persiste la brecha tecnológica y tenemos una nueva economía con altos niveles de dependencia, existen avances cualitativos que han facilitado las actividades productivas, hoy en día, podemos hablar de la conexión de todo tipo de dispositivo que posibilitan un nuevo espacio social para la interrelación e interacción de los agentes económicos, con ayuda de herramientas tecnológicas fuera de oficina sin horario, los negocios virtuales, mediante portales de compra y venta que cada vez se incrementan sus volúmenes de transacciones, la banca virtual donde, la reducción del tamaño de las empresas que es 
reemplazada por las MIPYMES, las áreas de negocios de servicios, que buscan satisfacer la comodidad de los clientes, que los servicios están sustentando mano de obra para satisfacer comodidades y no necesariamente la producción de bienes tangibles, que a la vez generan mayor inclusión social, rompiendo la visión instrumentalizada de las tecnologías, convirtiéndolas potenciadoras del desarrollo de esta nueva economía, pero que al mismo tiempo constituyen el inicio de un largo camino que falta recorrer.

\section{Conclusiones}

La esencia de la globalización debería estar marcada por un acceso, sin barreras y restricciones, así como propugna el sistema económico de libre mercado que sigue siendo hegemónico en la economía mundial; sin embargo, esto en la realidad no ocurre porque el acceso al conocimiento y al libre tránsito de la información esta mediado por el carácter privado cuyo propósito es la rentabilidad y el control del mercado, lo que lleva a señalar que el conocimiento no es un bien público, corresponde a la esfera privada lo que impide su globalización. Los países menos desarrollados son consumidores de bienes y servicios producidos por las naciones industrializadas, que acceden a la distribución y en algunos casos a la transferencia de tecnología, pero en condiciones altamente onerosas, lo que evidencia la brecha entre estos países, generando condiciones de dependencia que no promueve la competitividad y la innovación.

Para lograr el paso a una nueva economía, un elemento central es la calidad de la educación superior, que esté en capacidad de formar profesionales altamente competitivos en base a una oferta académica pertinente que responda a las demandas sociales, que promueva la investigación científica, la formación de investigadores con capacidad de innovar, destinando los recursos económicos necesarios para fortalecer esta función sustantiva de la universidad, dejando de lado los intentos de implantar modelos extranjeros ajenos a la realidad que únicamente se prestan para el desperdicio de recursos que de por si son escasos en la economía. Existen importantes avances en la utilización de las tecnologías de conectividad y telecomunicaciones, sin embargo, desafío es acortar la brecha tecnológica, profundizar los incentivos al emprendimiento como un factor fundamental en para alcanzar el desarrollo económico aprovechando el conocimiento y el talento humano para alcanzar una sociedad más equitativa y menos dependiente de los países desarrollados.

\section{Bibliografía}

Bizzozero, L. (2015). Emerging countries on the new map of the knowledge society. Cuestiones Políticas, 4-5.

Castells, M. (1999), La Sociedad Red, Vol. 1, México, Ed. Siglo XXI.

Castells, M. (2001). La era de la información: vol. 1. La sociedad red. Madrid: Alianza Editorial.

Darín, S.; González, Y. (2008). Nuevos paradigmas de la gestión en un cambio de época: de la era industrial a la era del conocimiento, los retos derivados de la globalización. Ciencias De La Información, 39(1), 53-58.

Drucker, P. (1969). La era de la discontinuidad. Información Comercial Española, 431, 15 - 34. 
Drucker, P. (1993). Administración y futuro. De los '90 en adelante, Buenos Aires, Editorial Sudamericana.

Drucker, P (1993). La sociedad poscapitalista, Barcelona, Apóstrofe.

Finquelievich, S. (2004). La sociedad civil en la economía del conocimiento: TICs y desarrollo socioeconómico. Instituto de Investigaciones Gino Germani.Facultad de Ciencias Sociales. UBA Buenos Aires. Argentina

Guevara, C. (2013). Economía del conocimiento y la innovación (ECI). Fundación Omar Dengo. Costa Rica.

Hargreaves, A. (2003). Teaching in the knowledge society. Education in the age of insecurity. Tecahers College Press.

Krüger, K. (2006). El concepto de 'sociedad del conocimiento'. Revista Bibliográfica de Geografía y Ciencias Sociales. Universidad de Barcelona. Vol. XI. Nro. 683.

Lamo de Espinosa, E. (2004) Bajo Puertas de Fuego. El Nuevo Desorden Internacional. Taurus. Madrid, España. 205 pp.

Nino, E. (2011). La desigualdad en el acceso a la educación universitaria argentina. Lecciones Y Ensayos, 89, 351-366.

Pérez, D., Núñez, I., \& Font, E. (2016). Globalización y desarrollo local, una propuesta metodológica de información y gestión del conocimiento, 157(Número 2), 107-120.

Rodríguez, E.; Palma, A. (2010). Desafíos de la educación superios en la economía del conocimiento. Revista Chilena de Ingeniería. Universidad de Tarapacá. Chile.

Rúa, N. (2006). La globalización del conocimiento científico tecnológico y su impacto sobre la innovación en los países menos desarrollados. UNED. España.

Senplades (2017). Plan para el buen vivir 2017 - 2021.Quito. Ecuador.

Stehr, N. (1994). Knowledge Societies. Sage.

Tobón, S., Guzmán, C. E., \& Cardona, S. (2015). Sociedad del conocimiento: estudio documental desde una perspectiva humanista y compleja. Revista Paradigma, XXXVI, 7-36.

UNESCO, (2005). Informe Mundial de la Unesco. Hacia las Sociedades del Conocimiento. Publicado en 2005 por la Organización de las Naciones Unidas para la Educación, la Ciencia y la Cultura

Villavicencio, A. (2013). ¿Hacia dónde va el Proyecto Universitario de la Revolución Ciudadana? Boletín Informativo Spondyllus. ASB. Quito. Ecuador. 